The meetings in this list are all organized by Divisions of EPS (boxed) or sponsored by EPS. Europhysics Study Conferences, described in Europhysics News 35 (June 1972), are marked by an $\Theta$ before the date. It is regretted that, because of the restriction on the amount of space for this section, it is not possible to give details of other meetings about which EPS has been notified. However, a full list of events is available in the Meetings Issue of Europhysics News 35 (June 1972).

The order of information is : date, title, venue, and contact for information.

\section{Conferences 1972}

25 - 28 July

Selected Problems in Magnetism

Bochum, Federal Republic of Germany

D. Wagner, Ruhr Universität Bochum,

Institut für Theoretische Physik III. Postfach 2148 ,

D.463 Bochum-Querenburg

21-25 August

5th European Conference on Controlled Fusion and Plasma Physics

Grenoble, France

T. Consoli, Département de la Physique du Plasma et de la Fusion Controlée, Service d'lonique Générale, Centre d'Etudes Nucléaires de Grenoble, Cédex 85, F-38 Grenoble-Gare

21- 26 August

17th Ampere Congress - Nuclear Magnetic

Resonance and Related Phenomena

Turku, Finland

Mrs P. Somerkoski, Conference Office,

17th Ampere Congress, University of Turku,

Wihuri Physical Laboratory,

20500 Turku 50

\section{8 - 31 August}

International Conference on Applications of the Mössbauer Effect

Ayeleth Hashahar, Upper Galilee, Israel

M. Pasternak, Soreq Nuclear Research Center, Doar Yavne

29 August - 1 September

5th International Symposium on Discharges and Electrical Insulation in Vacuum

Poznan, Poland

M. Goldman, Laboratoire de Physique des Décharges, Ecole Supérieure d'Electricité, 10. Avenue Pierre-Larousse,

F-92 Malakoff

\section{G September 1972}

Intermediate Processes in Nuclear Reactions Plitvice Lakes, Yugoslavia

P. Kulisic, Department of Physics, Faculty of Electrical Engineering, Zagreb, Unska b.b., P.O. Box 170
4- 10 September

International Symposium on the Physics and Technology of Semiconductor Light Emitters and Detectors

Pugnochiuso (Foggia), Italy

A. Frova, Istituto di Fisica, Università di Roma, Piazzale delle Scienze 5, I-000185 Roma

12 - 15 September

2nd European Solid State Device Research Conference (ESSDERC 1972)

Lancaster, UK

C. Hilsum, Royal Radar Establishment St. Andrews Road, Malvern, Worcs

18 - 21 September

Third European Symposium on Cosmic Rays Modulation Effects

Göttingen, Fed. Rep. of Germany

H.J. Müller, Max-Planck-Institut f. Aeronomie Postfach 60 D-3411 Lindau/Harz

18 - 25 Septembe

Symposium on the Development of the Physicists' Conception of Nature

Trieste, Italy

A. Salam, International Centre for Theoretical Physics, P.O. Box 586, I-34100 Trieste

G 28 -30 September

Colloquium On "Phonons"

L'Aigoual, France

Mlle A.M. Vergnoux, Laboratoire de Physique Moléculaire et Cristalline, Faculté des Sciences de Montpellier.

chemin des Brusses, F-34 Montpellier

\section{EPS General Conference}

3-6 October

Trends in Physics

Wlesbaden, Fed. Rep. Germany

U. Dihle, Physikalische Institut der Universität, Rober Mayer-Strasse 2-4, D-6000 Frankfurt/Main

\section{'Summer' schools 1972}

4-15 September

International Summerschool on Fusion Reactor Problems

Erice, Italy

B. Brunnelli, Laboratori Gas Ionizzati,

Eurotom-CNEN,

C.P. $65,1-00044$ Frascati

21 September - 4 October

Plasma Heating and Injection

Varenna, Italy

P. Caldirola, Laboratorio di Fisica del Plasma, I-20133 Milan Via Celoria 16

\section{Conferences 1973}

2-7 July

Lattice Deffects in Ionic Crystals Ile de Bandor (Var), France

M. Chemla, Laboratoire d'Electrochimie, Université de Paris VI.

9, Quai St-Bernard, F-75 Paris $5 e$
28 August - 1 September

1st Specialized Ampere Colloquium - NMR in Solids : Pulse Methods, High Resolution, Spin Dynamics and Related Phenomena

Cracow, Poland

J.W. Hennel, Instytut Fizyki Jadrowej ul Radzikowskiego 152, Cracow 23

10 - 14 September

3rd International Meeting on Ferroelectricity

Edinburgh, UK

W. Cochran, University of Edinburgh. Department of Physics, Mayfield

Road, Edinburgh EH9 3JZ

Conference and 'summer' school organizers who wish to have their meetings included in the next Meetings Issue of Europhysics News in December 1972 should apply to :

Main Secretariat,

European Physical Society

P.O. Box 39,

CH - 1213 PETIT-LANCY 2,

Switzerland.

The Main Secretariat will forward a questionnaire for completion and return.

\section{New address for EPS}

The Main Secretariat of the European Physical Society has moved to a new address. In future, all correspondence on EPS matters, as well as for Europhysics News, should be sent to:

\section{European Physical Society, P.O. Box 39, CH-1213 PETIT-LANCY 2 , Switzerland Telephone : Geneva 931130}

\footnotetext{
Editor : Alex H. Crawtord

Editorial Advisory Panel :

G.-J. Béné, L. Etienne, L. Jansen,

E.N. Shaw, B. Southworth.

All correspondence to : Editor, EUROPHYSICS NEWS

European Physical Soclety,

P.O. Box 39,

CH - 1213 Petit-Lancy 2

Phone : Geneva 931132

Switzerland.

Published by the European Physical Soclety

Printed by : Ed. Cherix et Fllanosa S.A. CH-1260 Nyon, Switzerland.
} 